\title{
Nano delivery of natural therapy for cancer treatment
}

\author{
A. Chakraborty ${ }^{1}$, B.G. Roy ${ }^{2}$, K.M.Waidha ${ }^{1}$ and S. S. Lahiri ${ }^{1 *}$ \\ ${ }^{1}$ Amity Institute of Biotechnology, Amity University, Noida, Uttar Pradesh -201301.India \\ ${ }^{2}$ Institute of Nuclear Medicine \& Allied Sciences, Delhi-110054 \\ *Email: sslahiri@amity.edu
}

Cancer Summit 2015 records the complete failure cancer therapy as on today, stating that the conventional chemotherapy cause more cancer than cure [1]. Besides the chemotherapeautic agents lack in specificity and affect both malignat as well as healthy cells alike. Selective killing of tumor cells, leaving the healthy cells unaffected, is the aim of all reasearch. The present invention provides an anti-cancer formulation incorporated in a nanoporous hydrogel, is able to selectively cause apoptosis of cancer cells keeping the normal healthy cells unharmed. The invention provides an anti-cancer formulation comprising of aqueous extract of the leaves of Belada mara (Aegle marmelos) (Figure 1) [2]; aqueous extract of the fresh leaves of the plant Oxalis corniculata [3]; aqueous extract of the cotyledons of the seed of custard apple (Annona reticulate) and aqueous extract of the seeds of Fenugreek (Trigonella foenum-graecum); Newcastle disease virus [4] suspended in $1 \mathrm{X}$ Phosphate buffer saline, aqueous extract of Ginseng from the plant Panax ginseng, all at declared proportions.Delivery of the above formulation is based on a hydrogel for oral delivery and slow release of this and a process for preparation thereof. The formulation comprises agarose and protein hydrolysate in phosphate buffer and saline with mixed Polyethelene Glycol and Glycerol. The hydrogel of the present invention is used as a carrier for oral delivery of drugs. The formulation studied, is free from any toxicity and components are consumed as food or for therapy by human. The hydrogel in the present invention can be made for targeted release either in the stomach or in the intestine by altering the buffer $\mathrm{pH}$ during the manufacture.

The anticancer formulation incorporated in the nano-porous hydrogel is able to selectively cause apoptosis to cancer cells (Breast cancer: MCF7, Cervical cancer: NCI60) and keeps the normal healthy cells (Fibroblast: DF1) unharmed. Cancer induced strain A mice were treated with the formulation with encouraging results (Figure 2). Survival of $100 \%$ cancer induced strain. Amice when treated with the formulation on visible signs of tumor. Delayed treatment resulted in $67 \%$ survival of mice with no change in tumor size or any further metastasis.

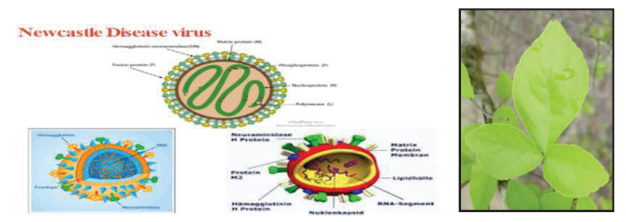

Figure 1: Newcastle disease virus(left) and Belada mara leaf (right)

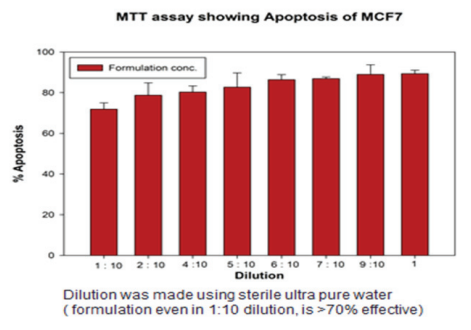

Figure 2: Results of MTT assay

Keywords: oncolytic virus, drug delivery, drug specificity, nanporous gel

\section{References}

1. Sun, Y. et.al. (2012). Treatment-induced damage to the tumor microenvironment promotes prostate cancer therapy resistance through WNT16B. Nature medicine, 18(9), 1359-1368.

2. Bhatti, R., et.al. (2013). Pharmacognostic standardisation and antiproliferative activity of Aegle marmelos (L.) Correa leaves in various human cancer cell lines. Indian Journal of Pharmaceutical Sciences, 75(6), 628-634.

3. Ashwani K et.al (2012) An Absolute Review on Oxalis corniculata Linn. Intl. J. of Res. in Pharma. and Biomed Sci. Vol. 3 (3), 1173-1188.

4. Zamarin, D., and Palese, P. (2012). Oncolytic Newcastle disease virus for cancer therapy: old challenges and new directions. Future microbiology, 7(3), 347-367. 